\title{
///IOMENTUM
}

QUARTERLY

ZEITSCHRIFT FÜR

SOZIALEN FORTSCHRITT

2020 | Vol. 9. No. 1, p. 4-17

\section{Die Auswirkungen von Kindergartengebühren auf die Erwerbstätigkeit von Frauen mit Kindern}

\author{
Felix Zangerl ${ }^{\star}$ und Isabel Pham ${ }^{* *}$
}

Zusammenfassung

Diese Arbeit befasst sich mit der Frage, inwieweit sich die Abschaffung von Kindergartengebühren auf die Erwerbstätigkeit von Frauen mit Kindern unter sechs Jahren auswirkt. Den Anstoß zur Arbeit bot die Abschaffung der Gebühren im Bundesland Wien im Herbst 2009. Für die Analyse wird eine „Difference in differences“-Schätzung durchgeführt, bei der Wien mit anderen urbanen Regionen in Österreich verglichen wird. Der Beobachtungszeitraum umfasst das Jahr vor (2008) und das Jahr nach (2010) der Gebührenabschaffung. Mit Daten aus dem Mikrozensus wird das Ausmaß an Wochenarbeitsstunden der Frauen durch sozioökonomische Faktoren erklärt. Da Frauenerwerbstätigkeit durch eine Vielzahl an (un)beobachtbaren Faktoren determiniert wird und Kinderbetreuung nur einen Teil zur Erklärung beiträgt, resultiert die empirische Analyse der Auswirkung der Gebühren auf die Frauenerwerbstätigkeit in keinen signifikanten Effekten.

Schlagwörter: Frauenerwerbstätigkeit, Kindergartengebühren, Arbeitsangebot

The effects of kindergarden fees on the labour market participation of women with children

\section{Abstract}

The presented analysis investigates how eliminated kindergarden fees influence the labour market participation of women with children under the age of six. The elimination of kindergarden fees in Vienna in autumn 2009 represents the initial motivation for the policy analysis. We conduct a "difference in differences" estimation by comparing Vienna to other similar urban areas in Austria, where the year before (2008) and after (2010) the policy change constitutes the time frame of analysis. We try to explain the extend of women's weekly working hours through various socioeconomic factors recorded in the Mikrozensus survey. As childcare only partially explains female labour market participation which is determined by many more (un)observed factors, we cannot claim that the empirical analysis of the fees' influence on female labour market participation yields significant effects.

Keywords: Female labour market participation, kindergarden fees, labour supply

${ }^{*}$ Felix Zangerl, Budgetreferent, Wirtschaftswissenschaft in der AK Wien. E-Mail: felix.zangerl@gmail.com

** Isabel Pham, Student Assistant, Health Economics and Health Policy, IHS. E-Mail:phamisabel@gmail.com 


\section{Einleitung}

Die vorliegende Forschungsarbeit untersucht, inwieweit der Zusammenhang zwischen Gebührenstrukturen von Kinderbetreuung und Frauenerwerbstätigkeit festgestellt werden kann. Die Orientierung erfolgt entlang der Forschungsfrage „Wie verändert sich das Wochenarbeitsstundenausmaß von Frauen mit Kindern, wenn sich Kinderbetreuungskosten verändern?".

Der Hintergrund dieser Arbeit liegt in momentanen sozialpolitischen Umstrukturierungen, in die auch die Wiedereinführung der Kindergartengebühren für Nachmittagsbetreuung in Oberösterreich mit Herbst 2018 fällt. Neun Jahre zuvor waren die Elternbeiträge mit der Kinderbetreuungsgesetz-Novelle 2009 zeitgleich in Wien und Oberösterreich abgeschafft worden (Land Oberösterreich o. D.). Damalige Zielsetzungen waren die „Weiterentwicklung des Kindergartens als Bildungseinrichtung " und die Vereinbarkeit von Familie und Beruf zu fördern.

Obwohl das Land Oberösterreich seit der Wiedereinführung der Nachmittagsgebühren im Kindergarten mit Anfang September 2018 Abmeldungen im dreistelligen Bereich verzeichnete (Kurier 2018), ist der unmittelbare Zusammenhang mit Entscheidungen über die Partizipation am Arbeitsmarkt nur hypothetisch skizzierbar. Wenngleich Einführung und Abschaffung von Gebühren nicht kongruente Effekte auf Arbeitsmarktpartizipation zugesprochen werden, eignet sich die Betrachtung der 2009 abgeschafften Elternbeiträge aufgrund der bereits erhobenen Daten besser für unsere Fragestellung.

Wenn Gebührenpolitik gewisse wirtschaftspolitische Ziele verfolgt, so war dies im Falle der Kinderbetreuungsgesetz-Novelle 2009 die Förderung der Vereinbarkeit von Familie und Beruf und elterlicher Berufstätigkeit (Land Oberösterreich o. D.). Dies ist auch im zukünftigen Interesse der EU-Arbeitsmarktpolitik, in deren Europa-2020-Strategie die Steigerung der Erwerbstätigkeit festgeschrieben ist. Eines der fünf Kernziele dieser Strategie ist die Anhebung der Beschäftigungsquote $^{1}$ für 20 - bis 64 -jährige Frauen und Männer auf 75 \%. Die letzte türkis-blaue Bundesregierung gab eine höhere Beschäftigungsquote von 77 bis $78 \%$ als Ziel vor. In Österreich sind bereits $74 \%$ der Männer und $70 \%$ der Frauen beschäftigt. Ob die festgesetzten Ziele erreicht werden, hängt im Wesent-

1 Zum Erhebungszeitpunkt Erwerbstätige. lichen davon ab, inwiefern eine Anhebung der Frauenbeschäftigungsquote möglich ist.

Bei Betrachtung des Erwerbstätigenanteils unabhängig vom Alter verzeichnet die Statistik Austria im Jahr 2018 63,7 \% aller Männer und 53,3 \% aller Frauen als erwerbstätig (Statistik Austria 2018a). Die Aufschlüsselung nach Stundengrenzen ergibt, dass 47,5\% aller Frauen und 11,2 \% der Männer teilzeitbeschäftigt sind (Statistik Austria 2018b).

Sowohl die ungleiche Stundenverteilung als auch die Quote an Erwerbstätigen geht mit der innerhäuslichen Verteilung familiärer Verpflichtungen einher (Haider et al. 2004). Sieht man sich individuelle Haushalte an, ist in heterosexuellen Partnerschaften die Wahrscheinlichkeit groß, dass die Frau überdurchschnittlich mehr an Reproduktionsarbeit (Kinderbetreuung, Haushalt, Pflege etc.) leistet. Während unsere Daten keine Intra-Haushalts-Informationen zur Rollenverteilung abbilden, wird in dieser Arbeit vom „male breadwinner model“ (siehe Kapitel 5 - Modell) ausgegangen.

Weiters beziehen sich die hier verwendeten Bezeichnungen „Frau“, „Mann“, „Vater“, „Mutter“ auf das im Mikrozensus erfasste Geschlecht unabhängig von der eigenen Geschlechtsidentität. Alternative Haushaltsmodelle, die von der Heteronormativität abweichen, werden hier ausgenommen.

\section{Aktueller Forschungsstand}

Es existiert bereits eine Vielzahl an wissenschaftlichen Publikationen, die sich mit der Arbeitsmarktpartizipation von Frauen, insbesondere Frauen mit Kindern, beschäftigen. Betrachtet man direkte Effekte, so bezeugen Blau und Tekin (2007) in ihrer Arbeit „The Determinants and Consequences of Child Care Subsidies for Single Mothers", dass eine Subvention der Kinderbetreuungskosten $\mathrm{zu}$ einem durchschnittlichen Anstieg der Frauenerwerbstätigkeit um 5 bis $11 \%$ führt. Aufgeschlüsselt nach Beschäftigungsausmaß sinkt demnach auch die Wahrscheinlichkeit, vollzeitbeschäftigt zu sein, gegenüber der Wahrscheinlichkeit, einer Teilzeittätigkeit nachzugehen, um die Hälfte (Haider et al. 2004). Klar ist, dass es einen Zusammenhang zwischen Kinderbetreuungszeiten, Teilzeitbeschäftigung und Erwerbseinkommen von Frauen mit Kindern gibt.

Die theoretische Überlegung dahinter ist, dass die Entscheidung, ob und wie viel eine Frau mit Kind am Arbeitsmarkt partizipiert, von multidimensionalen Faktoren geprägt ist. Eine Dimension, entlang welcher 
die Entscheidung fällt, sind Kinderbetreuungskosten: Wie hoch sind die zu erwartenden Mehrkosten einer ausgelagerten Kinderbetreuung, anstatt diese selbst zu übernehmen? Wie hoch ist der zum Einkommen relative Betrag an Kinderbetreuungskosten? Können Verwandte die Kinderbetreuung übernehmen? Stellt sich heraus, dass die Frau familiär eingebettet ist, kann dies die Wahrscheinlichkeit für eine Vollzeitberufstätigkeit signifikant erhöhen. Wenn es sich hierbei um gemeinsam im Haushalt lebende Verwandte handelt, ist deren gesundheitlicher Zustand ein zusätzlicher wesentlicher Faktor. Gemeinsam im Haushalt lebende pflegebedürftige Eltern senken die Wahrscheinlichkeit für eine Vollzeitbeschäftigung gegen null (Haider et al. 2004).

Ein weiterer wichtiger Aspekt ist die kulturspezifische Einbettung von bezahlter Arbeit und unbezahlter Arbeit (Reproduktionsarbeit oder Care-Arbeit). So seien laut Wel/Knijn (2006) drei Faktoren für die Aufteilung von Reproduktionsarbeit von besonderer Bedeutung: Der erste Faktor beschreibt ein rationales Konzept, in dem die bezahlte Arbeit im Haushalt umso gleicher verteilt ist, je gleicher auch die unbezahlte Arbeit verteilt wird. Der zweite Faktor beschreibt die Care-Kultur. Wenn diese stark dominiert, werden Frauen die Verantwortung für die Reproduktionsarbeit übernehmen. Der dritte Faktor ist ein sozialer Faktor oder Beziehungsfaktor. Wenn Uneinigkeit über die Aufteilung der unbezahlten Arbeit mit dem Partner herrscht, führt dies dazu, dass die Frau die unbezahlte Arbeit übernimmt (Wel/Knijn 2006). Im Falle der alleinigen Haushaltsführung führt die zusätzliche Belastung der Reproduktionsarbeit zu einer Reduktion bezahlter Arbeit (Haider et al. 2004).

Jedenfalls gehören zur kulturellen Einbettung auch die in einer Gesellschaft vermittelten Werte. Dominiert neben einer Care-Kultur zusätzlich die Rolle der Frau als „homemaker“, wird die gerechte Verteilung von Reproduktionsarbeit erschwert. Schlussendlich kommt es zu einem Zusammenstoß aus in der Jugend vermittelten Familienwerten und egalitären Werten zur Beteiligung am Arbeitsmarkt, die sich in einem inneren Konflikt, der auch als „mother’s guilt“ bekannt ist, manifestieren (Fortin 2005).

\section{Theoretische Überlegungen}

\section{Familienmodell}

Wie eingangs in der Einleitung erwähnt, gehen sowohl die Anzahl an Arbeitsstunden als auch die Quote der Erwerbstätigkeit mit der innerhäuslichen Verteilung familiärer Verpflichtungen einher (Haider et al. 2004). Während die empirische Analyse die unterschiedlichen Familienmodelle nicht unmittelbar berücksichtigt, setzt die Arbeit grundsätzlich am theoretischen Fundament des „male breadwinner model“ an. Das „male breadwinner model“ beschreibt die "traditionelle“ Rollenaufteilung in einem traditionellen Haushalt, ${ }^{2}$ wonach der Mann für die Lohnarbeit zuständig ist, sprichwörtlich das Brot nach Hause bringt, während die Frau „homemaker“ ist und die Reproduktionsarbeit erledigt. Dies umfasst sämtliche unbezahlte Arbeit, die notwendig ist, um sich selbst und den Nachwuchs zu ernähren und um den Haushalt zu führen; sich selbst und die eigene soziale Klasse zu reproduzieren (Fortin 2005). Schätzungsweise die Hälfte aller Familien in Österreich leben nach dem „male breadwinner model“ (Klapfer 2010, zitiert nach Berghammer 2014).

Beim „moderate breadwinner model“ (Wel/Knijn 2006) oder „modernized male breadwinner model“ (Berghammer 2014), welchem laut Wel/Knijn (2006) bereits $53 \%$ aller Mütter in einer in den Niederlanden durchgeführten Untersuchung angehören, arbeitet der Mann Vollzeit und die Frau Teilzeit. Diese Art der haushalterischen Arbeitsteilung wird auch unter dem Stichwort „neo-traditional family type“ beschrieben. In Österreich sind 47,5\% aller Frauen teilzeitbeschäftigt, während bei Männern die Teilzeitbeschäftigungsquote unter $12 \%$ liegt $^{3}$ (Statistik Austria 2018c). Unionsweit liegt Österreich mit dem Anteil an Teilzeitbeschäftigung an zweiter Stelle (Eurostat 2017).

Trotz allem ist ein allgemeiner positiver Langzeittrend zum „dual breadwinner model“ zu verzeichnen, bei dem sowohl Männer als auch Frauen Vollzeit berufstätig sind (Berghammer 2014). Im Wesentlichen sind finanzielle Unabhängigkeit und Selbstbestimmtheit positive Pfähle dieses Modells. Dies ist aber unweigerlich damit verknüpft, wie die Reproduktionsarbeit im Haushalt verteilt ist. Ist die Frau im „dual breadwinner model“ die Hauptverantwortliche für Reproduktionsarbeit, kommt es zu einer Doppelbelastung auf Kosten der bezahlten Arbeit. Berghammer (2014) analysiert auch die Auswirkung von Bildung auf die Arbeitsteilung innerhalb österreichischer Familien anhand von Paneldaten von 1980 bis 2009 und kommt zu dem Schluss, dass speziell bei jungen Frauen mit hohem Bil-

2 Der traditionelle Haushalt umfasst ein gemeinsam lebendes verheiratetes oder unverheiratetes heterosexuelles Paar mit Kindern.

3 Jahresdurchschnitt 2018 
dungsniveau wieder eine Rückkehr zum „male breadwinner model“ zu verzeichnen ist. Dies sei dadurch zu erklären, dass die Bildungsrendite ${ }^{4}$ in den letzten Jahren rückläufig und dadurch ein kurzfristiger Rückfall in traditionelle Rollen zu beobachten sei, der den langfristig positiven Trend hin zum „dual breadwinner model“ schwäche. Sieht man sich folglich den Zusammenhang zwischen Reproduktionsarbeit und Arbeitsmarktpartizipation an, kann aufgrund des vorhin skizzierten vorherrschenden „male breadwinner“-Modells von einem negativen Effekt auf die Wochenarbeitszeit von Frauen mit Kindern ausgegangen werden.

\section{Intrahaushaltsverteilung}

Das etablierte Familienmodell hat unmittelbare Auswirkung auf die tatsächliche Verteilung der Reproduktionsarbeit innerhalb des Haushaltes, was mittelbar, wie die Literatur eindeutig zeigt, die Erwerbstätigkeit von Frauen stark beeinflusst.

\section{Verwandte}

Eine Komponente, die in die Intrahaushaltsverteilung von Reproduktionsarbeit hineinspielt, wurde bereits in der Literatur angeführt: Die Bereitschaft der erweiterten Familie (z. B. Großeltern), notwendige Reproduktionsarbeit zu übernehmen, kann zur Entlastung der Kernfamilie beitragen und einen positiven Effekt auf die Wochenarbeitsstunden der Frau haben. Allerdings ist diese Bereitschaft von mehreren Faktoren, wie z. B. Gesundheitszustand, tatsächliche Entfernung (z. B. wohnhaft innerhalb des gemeinsamen Haushaltes) oder Beziehung zueinander, abhängig (Haider et al. 2004).

\section{Familienstand}

Ganz wesentlich für den Zusammenhang zwischen Familienstand und Arbeitsmarktpartizipation ist wieder die Grundannahme des „male breadwinner“Modells, dem die Hälfte aller in einem gemeinsamen Haushalt lebenden Familien in Österreich folgt (Klapfer 2010, zitiert nach Berghammer 2014). Die häusliche Partnerschaft und in weiterer Folge die Ehe als gesellschaftliche Institution verfestigt traditionelle Rollenbilder, z. B. durch die steuerliche Bevorzugung verheirateter Paare mit Kindern, Subventionen und rechtlichen Besserstellung, wie es in Deutschland

4 Bildungserträge, die im ökonomischen Kontext als monetäre Vergütung von Bildungsinvestitionen verstanden werden. noch aktuell der Fall ist (Dingeldey 200o). Vor dem Hintergrund der Existenz eines monetär abgesicherten Partners hängt die Arbeitsmarktpartizipation der verheirateten Frau im Wesentlichen vom vorherrschenden Familienmodell ab. Alleinerziehung konstituiert die zweite Ausprägung, die mittelbar über die Reproduktionsarbeit negative Effekte auf die Arbeitsmarktpartizipation hat. Es wird davon ausgegangen, dass Alleinerziehende am stärksten der Doppelbelastung von Lohnarbeit und Reproduktionsarbeit ausgesetzt sind. Wie im Literaturteil bereits argumentiert, wird davon ausgegangen, dass im Falle der alleinigen Haushaltsführung die zusätzliche Belastung der Reproduktionsarbeit zu einer Reduktion bezahlter Arbeit führt.

\section{Kinderbetreuungskosten}

Bei Kindergartengebühren sind heterogene Effekte auf die Anzahl an Arbeitsstunden zu erwarten. Zieht man das klassische Arbeitsmarktmodell heran, unternimmt das Individuum grundsätzlich bei der Entscheidung, wie viel es arbeitet, eine Allokation zwischen den Gütern Freizeit und Konsum. Konsum wird durch erhöhtes Arbeitsaufkommen ermöglicht, Freizeit durch dessen Reduktion. Unbezahlte Reproduktionsarbeit fällt in diesem Modell unter Freizeit. Kommt es jetzt bei gleicher Ressourcenausstattung zu einer Erhöhung des verfügbaren Einkommens, beispielsweise durch die Senkung der Kindergartengebühr, tritt der sogenannte negative Einkommenseffekt (vgl. Borjas/Van Ours 2000, 36 - siehe Abbildung 5 im Appendix) auf: Ein höheres verfügbares Einkommen führt dazu, dass der marginale Nutzen des Lohns einer zusätzlichen Stunde Arbeit gegenüber einer zusätzlichen Stunde Freizeit sinkt. Das Individuum „konsumiert" mehr Freizeit. Wenn eine Reduktion der Kinderbetreuungskosten daher als höheres verfügbares Einkommen verstanden wird, kommt es laut klassischer Arbeitsmarkttheorie zur Reduktion von Arbeitsstunden.

Dem entgegengesetzt existieren zahlreiche Publikationen, die einen positiven Zusammenhang zwischen der Reduktion von Kinderbetreuungskosten und der erhöhten Erwerbspartizipation von Frauen sehen. Dies hängt damit zusammen, dass bei der Abschaffung der Gebühr Frauen ihre Kinder eher in den Kindergarten geben und im Zuge dessen ihr Arbeitsangebot steigern. Abhängig davon, welcher der Effekte überwiegt, sind daher heterogene Effekte der Reform zu erwarten.

5 Dies passiert natürlich nicht unabhängig vom Ausgangsniveau der geleisteten Wochenarbeitsstunden. 
Abbildung 1: Zusammenhang zwischen Modellvariablen und Frauenerwerbstätigkeit

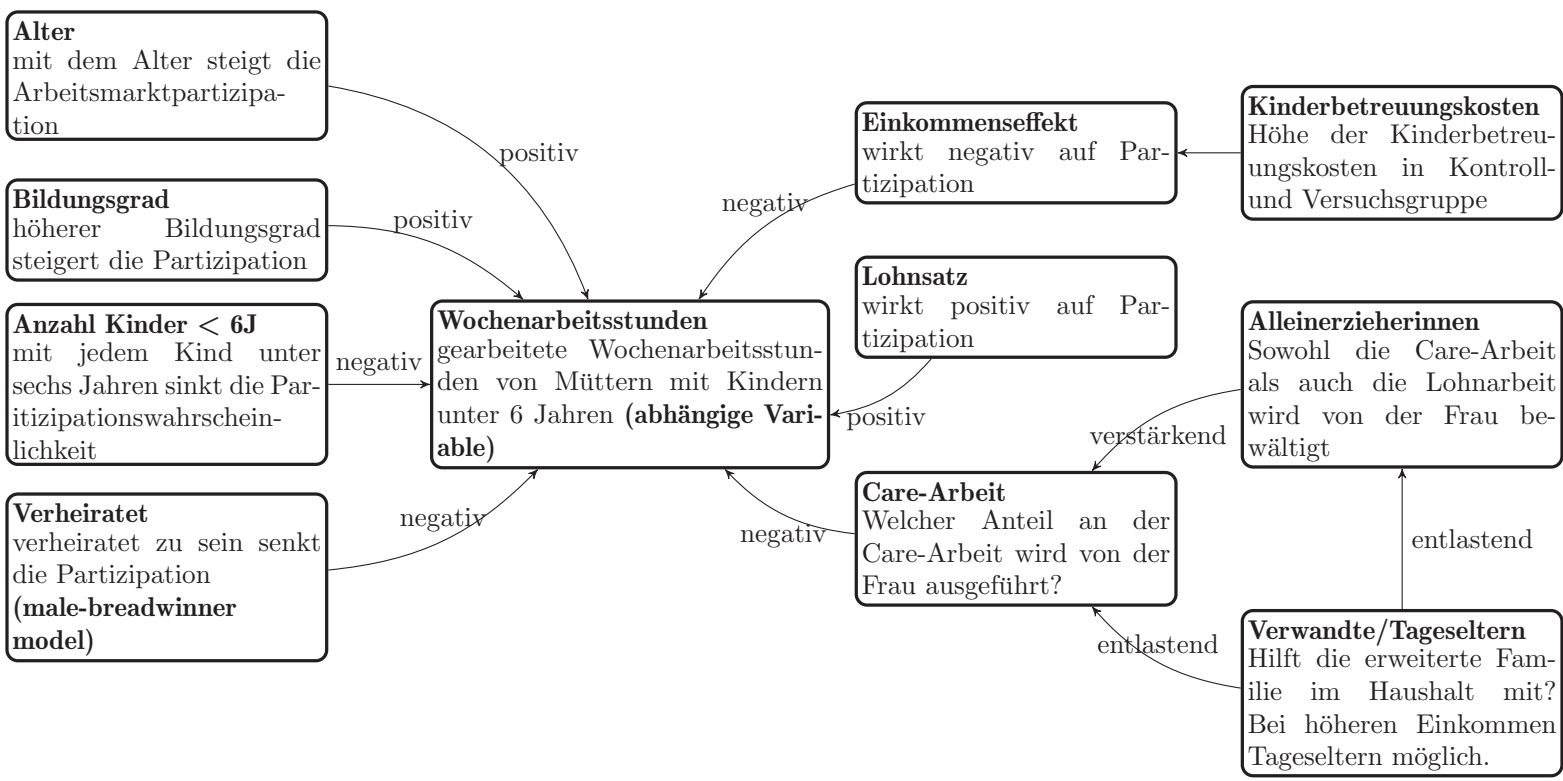

Quelle: eigene Darstellung

Alter, Bildung und Lohnsatz

In Bezug auf Alter und Bildung ist der Zusammenhang mit dem Arbeitsangebot in Form von Arbeitsstunden deutlicher: Wie in Abbildung 1 zu sehen, wird für das Alter ein positiver Effekt auf die Anzahl an Arbeitsstunden angenommen. Zunehmende Bildung weist aus Sicht der klassischen Humankapitaltheorie (Borjas/ Van Ours 2000) ebenso einen positiven Effekt auf. Dies ergibt sich aus der Annahme, dass Bildung Produktivität erhöht und Produktivität entlohnt wird. In der Erwartung, dass eine längere Ausbildungszeit, die mit Kosten verbunden ist, zukünftig höhere Bildungserträge abwirft, wird eine zukunftsorientierte Person, die viel Zeit in Bildung investiert hat, die Arbeitsmotivation zum Teil aus den entstandenen Kosten und erwartbaren Erträgen schöpfen. Bildung beeinflusst daher implizit über den Lohnsatz die Wochenarbeitszeit.

\section{Anzahl an Kinder}

Mit steigender Kinderanzahl nimmt die Wahrscheinlichkeit ab, am Arbeitsmarkt zu partizipieren. Jedes zusätzliche Kind erhöht den Gesamtaufwand im Haushalt. Da das zweite Kind eine andere Belastung darstellt als das fünfte und die Ausgangssituation vor jedem zusätzlichen Kind unterschiedlich ist, kann hier ein nicht-linearer Zusammenhang angenommen werden (Matysiak 2011).

\section{Modell}

Mit den vorhergehenden theoretischen Annahmen zum herrschenden Familienmodell sowie zur Intrahaushaltsverteilung der Reproduktionsarbeit ist das Ziel der vorliegenden Abbildung 1, die in der empirischen Analyse verwendeten Einflussfaktoren auf die Erwerbstätigkeit von Frauen darzustellen und zu skizzieren, wie sich deren Effekte auf die wöchentlichen Arbeitsstunden auswirken werden.

Das Kernmodell setzt sich zusammen aus den durchschnittlichen Wochenarbeitsstunden (abhängige Variable) und den erklärenden Variablen Alter, höchste abgeschlossene Ausbildung (Bildungsgrad), Anzahl der Kinder unter sechs Jahren und Familienstand.

Die Angebotsliteratur unterscheidet üblicherweise die extensive Margin, d. h. ob eine Person am Arbeitsmarkt partizipiert oder nicht, von der intensiven Margin, also der Anzahl der Arbeitsstunden, die angeboten wird. Für diese Arbeit wird nach Voranalyse, welche ergab, dass die Erwerbsbeteiligung (extensive Margin) nicht von der Gebührenabschaffung betroffen ist, der Fokus auf die intensive Margin, die Anzahl an Arbeitsstunden, als abhängige Variable gelegt.

Das erweiterte Modell bildet zusätzlich alle anderen Einflussfaktoren wie die durchschnittlichen 
Kosten der Kinderbetreuung, die Verteilung der Reproduktionsarbeit im Haushalt, Alleinerziehung, die Verfügbarkeit und Hilfsbereitschaft der erweiterten Familie, das Jahr der Untersuchung und den Wohnsitz der Person ab, um ein möglichst ganzheitliches Bild der Wechselwirkungen darzustellen.

Nicht alle Einflussfaktoren werden in der empirischen Analyse verarbeitet. Ziel der Modellschätzung ist es jedoch, für eine Frau mit durchschnittlichen Charakteristika (Alter, Bildung etc.) die durchschnittliche Wochenarbeitszeit zu prognostizieren. Um den Effekt der Gebührenabschaffung zu messen, werden Frauen, die davon betroffen sind, jenen gegenübergestellt, die nicht davon betroffen sind.

Für die Analyse werden nur Frauen aus urbanen Gebieten (siehe Kapitel 6 - Daten und Variablen) in die Untersuchung einbezogen. Dadurch wird sichergestellt, dass die institutionellen Faktoren wie Öffnungszeiten, Erreichbarkeit und Betreuungsquoten untereinander vergleichbar sind.

Zusammengefasst ergibt die vorherige Modellierung der Interaktion von Arbeitsmarktpartizipation und verschiedenen erklärenden Variablen folgende Forschungsfrage: „Wie verändert sich das Ausmaß an Wochenarbeitsstunden von Frauen mit Kindern, wenn sich Kinderbetreuungskosten verändern?"

Daraus kann folgende Hypothese abgeleitet werden:

$\mathrm{H}_{\mathrm{o}}$ : Frauen mit Kindern, die von einer Senkung der Kindergartengebühren betroffen sind, bringen mehr Wochenarbeitsstunden auf als nicht betroffene Frauen.

Die für die Modellschätzung relevanten Variablen werden im Kapitel 5 - Daten und Variablen ausführlich behandelt.

\section{Daten und Variablen}

Die Analyse beruht auf den Daten der Mikrozensus-Erhebung, einer Befragung in Österreich ansässiger Individuen und Haushalte zur Erwerbstätigkeit. Aufgrund der Verfügbarkeit von Informationen auf der Mikroebene liefert der Mikrozensus wichtige Erkenntnisse zur Soziodemografie in Österreich.

Pro Jahresquartal werden rund 22.00o Haushalte bzw. 45.000 Personen in Österreich befragt. Grundsätzlich wird jede Person/jeder Haushalt insgesamt fünf Mal im Abstand von drei Monaten befragt, um unterjährige Veränderungen abbilden zu können. Jede Einheit bleibt daher für mindestens ein Jahr im
Sample. Das Auswahlverfahren zur verpflichtenden Teilnahme ${ }^{6}$ geschieht via Zufallsprinzip aus dem Zentralen Melderegister (ZMR) (Statistik Austria 2018b).

Der von der Statistik Austria zur Verfügung gestellte Datenauszug beinhaltet über 9.00o Beobachtungen und 220 Variablen jeweils für das Jahr 2008 und 2010. Diese zwei Untersuchungsperioden wurden gewählt, um die Unterschiede vor und nach der PolicyReform, konkret die Abschaffung der Kindergartengebühren in Wien im Herbst 2009, aufzuzeigen.

Das gewählte Sample beschränkt sich auf in Österreich lebende Frauen mit Kindern unter sechs Jahren. In die Treatmentgruppe, jene Gruppe, die von der Abschaffung der Kindergartengebühren betroffen ist, werden Frauen mit Kindern unter sechs Jahren einbezogen, die für den Analysezeitraum in Wien wohnhaft sind. Frauen mit Kindern unter sechs Jahren, die nicht in Wien wohnhaft sind, werden als Kontrollgruppe herangezogen. Die Anzahl der Beobachtungen wird zusätzlich dadurch eingeschränkt, dass nur Frauen mit Kindern unter sechs Jahren, die in dicht besiedelten Gebieten wohnhaft sind, ${ }^{7}$ einbezogen werden. Urbane Gebiete in Oberösterreich, die aufgrund der mit Wien zeitgleichen Abschaffung der Kindergartengebühren im Herbst 2009 grundsätzlich Teil der Treatmentgruppe wären, werden aufgrund der geringen Fallzahl gänzlich aus der Analyse exkludiert.

Insgesamt besteht die Treatmentgruppe daher aus Beobachtungen in urbanen Gebieten Wiens. Die Kontrollgruppe setzt sich aus Beobachtungen in urbanen Gebieten aller anderen Bundesländern exklusive Oberösterreich zusammen.

Die detaillierte Aufbereitung und Bereinigung wird folgend beschrieben.

\section{Abhängige Variable}

Wochenarbeitsstunden

Die normalen Wochenarbeitsstunden entsprechen der Normalarbeitszeit, die die befragte Person im Interview angibt. Im Gegensatz dazu wird zusätzlich die tatsächliche Wochenarbeitszeit erfasst. Die Differenz der beiden Variablen ergibt die geleisteten Überstunden

6 Alle volljährigen Angehörigen eines in der Stichprobe einbezogenen Haushaltes sind laut der Erwerbs- und Wohnstatistikverordnung zur Auskunftserteilung verpflichtet.

7 Laut Eurostat-Definition sind das Flächenraster mit einer Bevölkerungsdichte von mindestens 1.500 Einwohner/innen pro km und einer Mindestpopulation von 50.000 (Eurostat 2018). 
Abbildung 2: Durchschnittliche Wochenarbeitsstunden von Frauen mit Kindern

Durchschnittliche Wochenarbeitsstunden von Müttern nach Region

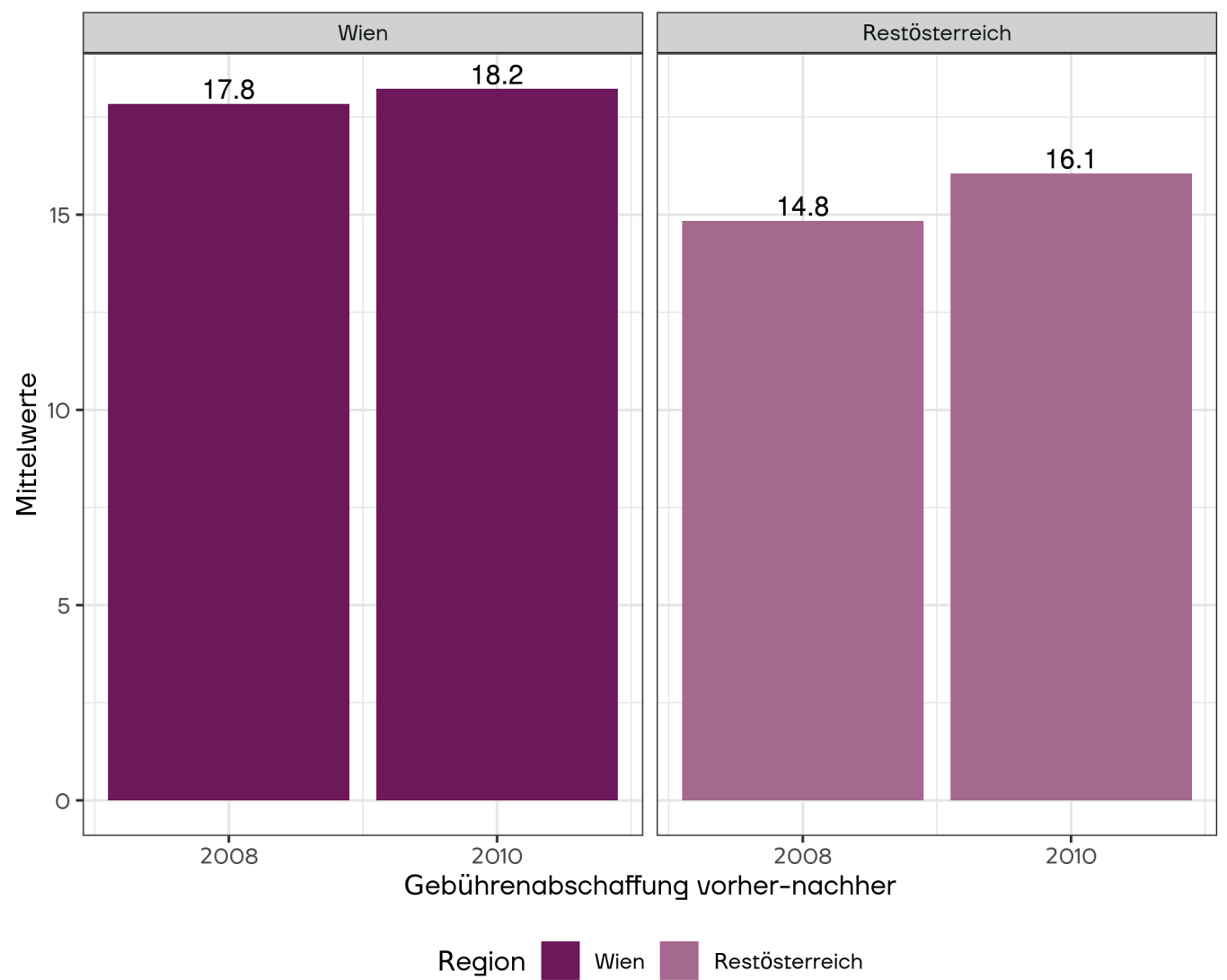

Quelle: Mikrozensus, eigene Berechnungen

bzw. entfallene Arbeitszeit aufgrund von Krankheit, Urlaub, Karenz, Mutterschutz etc. Normalarbeitszeit ist für diese Arbeit die bessere Wahl, da die Variable weniger Schwankungen aufweist, die durch Umstände wie z. B. Krankheit oder Urlaub ausgelöst werden, welche für die Analyse nicht relevant sind.

Die Variable ist numerisch und liegt zwischen o und 50 Stunden. Ausreißer, in diesem Fall Personen, die widersprüchliche bzw. unrealistische Angaben gemacht haben, wurden aus dem Datensatz genommen.

Um einen ersten Eindruck zu erhalten, wie die Ergebnisse unserer Untersuchung aussehen könnten, lohnt es sich, einen Blick auf die Verteilung und Mittelwerte der abhängigen Variable zu werfen. Ein erster Blick auf die Mittelwerte der Wochenarbeitsstunden von Frauen gruppiert nach Regionen in Abbildung 2 zeigt bereits, welche Ergebnisse in der Inferenzstatistik zu erwarten sind. Frauen, die in Wien leben, haben ihre Arbeitsstunden seit der Abschaffung der Kindergartengebühren unabhängig von allen Modellfaktoren um o,4 Stunden erhöht.
Die deutlich stärkere Arbeitsstundenerhöhung in den restlichen Bundesländern lässt vermuten, dass die Effekte multidimensional sind und nicht nur auf die Elternbeiträge zurückzuführen sind. Wichtig ist, auch die Ausgangssituation der Untersuchung zu betrachten: Abbildung 3 zeigt die Verteilung der gearbeiteten Wochenarbeitsstunden von Frauen gruppiert nach Region in absoluten Häufigkeiten. Der erste rote Balken beinhaltet 22 Frauen mit Kindern, die in Wien leben und nicht in Beschäftigung sind. In vielen Modellen würde eine unverhältnismäßig hohe Anzahl von nicht erwerbstätigen Personen im Sample ein Problem darstellen. Da hier allerdings nur die Differenz zwischen den zwei Untersuchungsjahren eine Rolle spielt, stellt die Ausgangsverteilung kein Problem dar. Wie sich anhand der Verteilung der Daten zeigt, sind Frauen mit Kindern in Wien deutlich seltener nicht erwerbstätig, wobei Vollzeitarbeit knapp überwiegt. In Restösterreich ist die überwiegende Mehrheit der Frauen nicht in Beschäftigung, während unter den Beschäftigten Teilzeitarbeit deutlich überwiegt. 
Abbildung 3: Verteilung der Wochenarbeitsstunden von Frauen mit Kindern im Jahr 2008

\section{Verteilung der Wochenarbeitsstunden von Müttern in 2008}

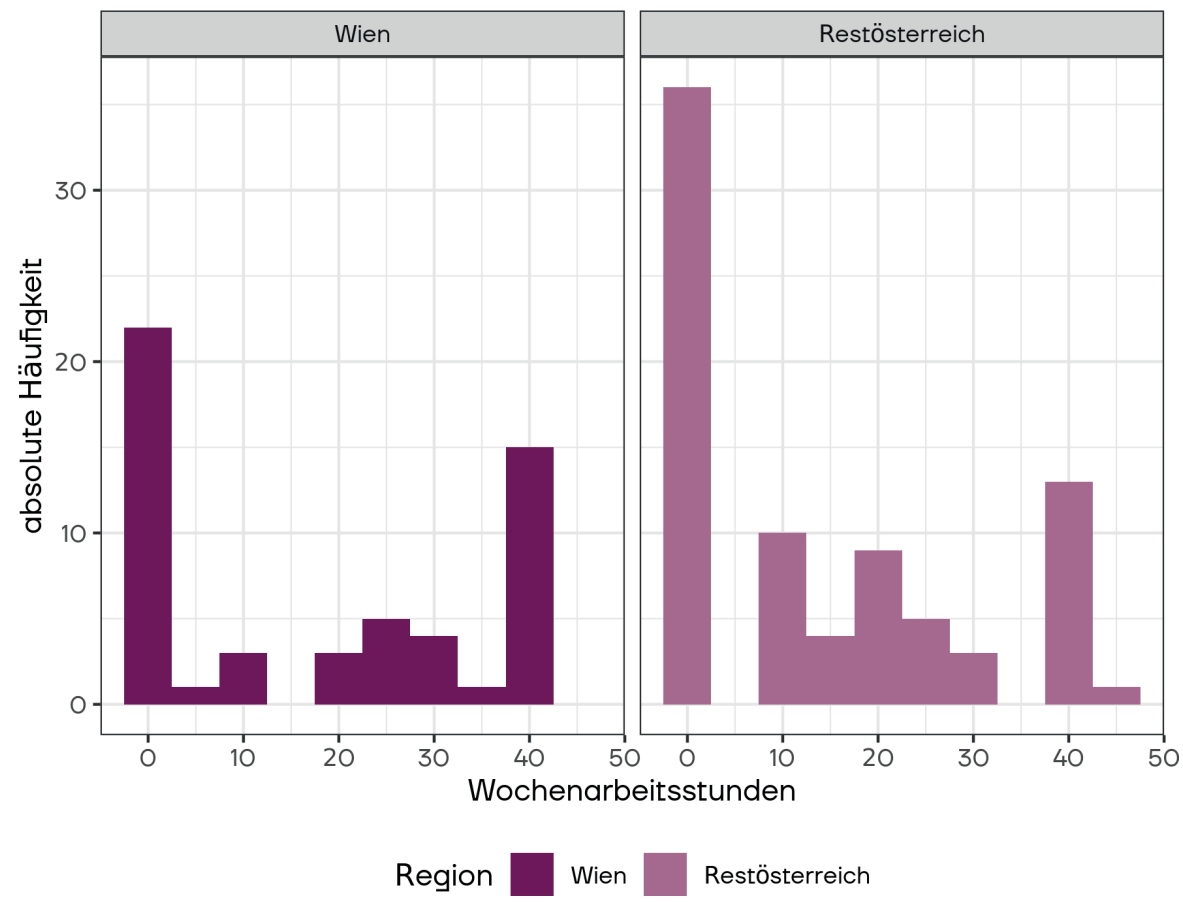

Quelle: Mikrozensus, eigene Berechnungen

\section{Erklärende Variablen}

Jahr - Wien: Die Variable ajahr bezeichnet das Jahr, in dem die Mikrozensusbefragung durchgeführt wurde. Die Variable xnuts2 gibt Aufschluss über den Haushaltsstandort der befragten Person, der mit der Variable xurb12 auf urbane Gebiete eingegrenzt wird. Der von Eurostat entwickelte und europaweit einheitliche Standard der NUTS-Klassifizierung entspricht weitgehend den nationalen territorial-administrativen Grenzen. Auf Ebene der NUTS2-Codierung entspricht die Klassifizierung den österreichischen Bundesländern. Die Variable xurb12 hat drei Ausprägungen, wobei nur die niedrigste Zahl ( 1 = dicht besiedelte Regionen) für die Untersuchung relevant ist. Die Interaktion der beiden Variablen ajahr $\times$ xnuts 2 ist zentral für die Difference in differences-Schätzung. Auf die genaue Interpretation wird im Kapitel Methodik eingegangen.

Alter: Das Alter der befragten Frauen mit Kindern wird im Mikrozensus in 15 Alterskategorien zusammengefasst. Jede Kategorie umfasst fünf Jahre ab dem 15. Lebensjahr, während o-14-Jährige eine eigene Kategorie bilden. Die Bildung der Mittelwerte in den jeweiligen Alterskategorien ermöglicht eine numerische Weiterverarbeitung. Die Mittelwerte reichen von 17 bis 47 Jahre.
Bildungsgrad: Der höchste abgeschlossene Bildungsgrad entspricht der österreichischen Klassifizierung von Ausbildung. Zwecks Übersichtlichkeit werden ähnliche Ausbildungskategorien zusammengefasst. Die modifizierte Variable ist eine Faktorvariable mit vier Ausprägungen:

- Pflichtschule

- Lehre (Lehrabschluss/BMS)

- Matura (AHS/BHS/BHS-Abiturientenlehrgang, Kolleg)

- Universität/Fachhochschule (Universitätslehrgänge, Universität, Fachhochschule, Hochschulverwaltete Lehrausbildung)

Anzahl an Kindern: Die Anzahl der Kinder im Haushalt wird numerisch erfasst. Das Sample inkludiert nur jene Frauen im modifizierten Sample, die mindestens ein Kind unter sechs Jahren haben.

Familienstand: Um zu messen, welchen Effekt der Familienstand auf die wöchentlichen Arbeitsstunden der Frau hat, wird eine Dummy-Variable erzeugt, die den Wert 1 annimmt, wenn die Frau im Sample verheiratet ist. 
Tabelle 1: Übersicht über die verwendeten Variablen

\begin{tabular}{|c|c|c|c|c|c|c|}
\hline Variable & $\mu$ & SD & Median & Min & Max & $\mathrm{N}$ \\
\hline Alter & 32,37 & 6,71 & 32,00 & 17,00 & 47,00 & 273,00 \\
\hline Stunden (gesamt) & 16,43 & 15,87 & 15,00 & 0,00 & 45,00 & 273,00 \\
\hline \multicolumn{7}{|c|}{ Stunden nach Kategorie } \\
\hline Pflichtschule & 9,53 & 15,21 & 0,00 & 0,00 & 45,00 & 83,00 \\
\hline BMS/Lehre & 18,99 & 15,67 & 20,00 & 0,00 & 45,00 & 91,00 \\
\hline Matura/Kolleg & 16,34 & 14,84 & 16,00 & 0,00 & 40,00 & 58,00 \\
\hline Universität & 21,73 & 15,08 & 20,00 & 0,00 & 45,00 & 65,00 \\
\hline 1 Kind & 20,06 & 16,08 & 20,00 & 0,00 & 45,00 & 127,00 \\
\hline 2 Kinder & 15,44 & 15,09 & 15,00 & 0,00 & 42,00 & 94,00 \\
\hline 3 Kinder & 12,71 & 15,26 & 1,00 & 0,00 & 40,00 & 57,00 \\
\hline 4+Kinder & 8,24 & 14,83 & 0,00 & 0,00 & 40,00 & 19,00 \\
\hline Wien & 18,04 & 16,83 & 20,00 & 0,00 & 45,00 & 112,00 \\
\hline Verheiratet & 15,19 & 15,41 & 12,00 & 0,00 & 45,00 & 202,00 \\
\hline Nicht Verheiratet & 19,07 & 16,58 & 20,00 & 0,00 & 45,00 & 95,00 \\
\hline Erwerbstätig & 26,52 & 11,76 & 25,00 & 1,00 & 45,00 & 184,00 \\
\hline Nicht Erwerbstätig & 0,00 & 0,00 & 0,00 & 0,00 & 0,00 & 113,00 \\
\hline
\end{tabular}

Quelle: Mikrozensus, eigene Berechnungen

Staatsbürgerschaft: Die Staatsbürgerschaft ist eine Faktorvariable mit acht Ausprägungen und wird zusätzlich als Kontrollvariable ins Kernmodell mitaufgenommen:

- Österreich

- EU-15 (ohne Österreich)

- EU-25 (zehn zusätzliche Länder ab 2004)

- Ex-Jugoslawien (ohne Slowenien, Kroatien)

- Türkei

- Sonstige

- Bulgarien, Rumänien (EU-27 ab 2007)

- Kroatien (EU-28 ab Juli 2013)

Tabelle 1 stellt eine Zusammenfassung der in der Schätzgleichung enthaltenen Variablen dar. Für die ersten zwei Variablen wird die Statistik der entsprechenden Variable dargestellt. Für die restlichen Variablen werden die Wochenarbeitsstunden für die jeweilige Gruppe ausgegeben.

\section{Methodik}

Wir wollen nun, wie bereits dargelegt, folgende Hypothese testen:

$\mathrm{H}_{\mathrm{o}}$ : Frauen mit Kindern unter sechs Jahren, die von einer Senkung der Kindergartengebühren betroffen sind, haben eine höhere Wahrscheinlichkeit, ihre Wochenarbeitsstunden zu erhöhen als nicht betroffene Frauen.

Die beste Herangehensweise, um den Effekt einer Policy-Reform zu messen, ist gemäß dem Stand der Forschung die Difference in differences (DID)-Schätzung. Diese kann mit der vorhandenen Datenstruktur, jeweils zwei Cross Sections für die Jahre 2008 und 2010, die zusammengefasst eine Pooled Cross Section ergeben, durchgeführt werden. Hiermit ist es möglich, Effekte festzustellen, die sich über die Zeit verändern, während über die Zeit gleichbleibende Effekte isoliert werden. Die Policy-Reform kann als „natürliches Experiment“ (Wooldridge 2016) aufgefasst werden, indem die beteiligten Individuen aufgrund von nicht durch den/die Forscher/in kontrollierbaren Ereignissen in Kontroll- und Treatmentgruppe eingeteilt werden. Genauso wie bei einem kontrollierten Experiment kann aber auch die Reliabilität von Hypothesen mithilfe von Signifikanztests geprüft werden.

Das Sample wird daher in vier Gruppen geteilt: Kontrollgruppe vor und nach der Reform, Treatmentgruppe vor und nach der Reform. Die Treatmentgruppe setzt sich zusammen aus Frauen mit Kindern unter sechs Jahren, die in Wien wohnhaft sind. Die Kontrollgruppe sind Frauen mit Kindern unter sechs Jahren, die nicht in Wien wohnhaft sind. 


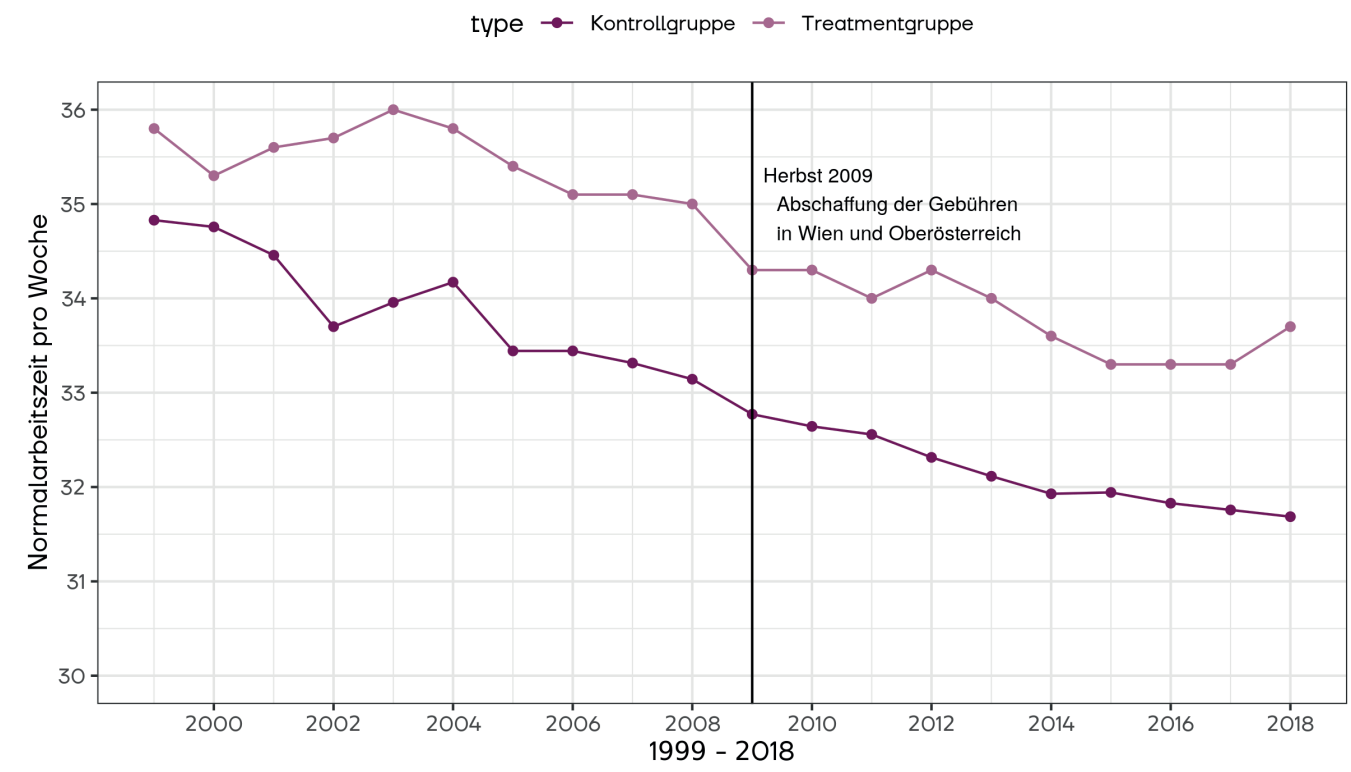

Quelle: Eurostat (2018), eigene Berechnungen

Der wesentliche Effekt $\delta_{1}$ (siehe Schätzgleichung 1), auch „average treatment effect" genannt, bildet den durchschnittlichen Effekt der Reform auf die y-Variable ab. Dieser setzt sich zusammen aus der durchschnittlichen Differenz ( $\bar{y})$ zwischen der Treatment (T)- und Kontrollgruppe (C) in Abhängigkeit von der Zeit $(\mathrm{t}): \hat{\delta}=\left(\overline{\mathrm{y}}_{\mathrm{t}=1, \mathrm{~T}}-\overline{\mathrm{y}}_{\mathrm{t}=1, \mathrm{C}}\right)-\left(\overline{\mathrm{y}}_{\mathrm{t}=0, \mathrm{~T}}-\overline{\mathrm{y}}_{\mathrm{t}=0, \mathrm{C}}\right)$

\section{Schätzgleichung}

Die Schätzgleichung lautet daher: $y=\beta_{0}+\delta_{o} D t+$ $\beta_{1} \mathrm{DT}+\delta_{1} \mathrm{Dt} \times \mathrm{DT}+$ andere Faktoren $+\varepsilon(1)$

wobei

- y: wöchentliche Arbeitsstunden,

- Dt (Zeitdummy): o für 2008 und 1 für 2010,

- DT (Treatmentdummy): o, wenn Person nicht in Wien lebt; 1, wenn Person in Wien lebt,

- Dt $\times$ DT (Interaktionsterm): Der Koeffizient des Interaktionsterms lässt sich interpretieren als die Differenz zwischen den Mittelwerten der beiden Jahre 2008, 2010 und der beiden Standorte (Wien und Restösterreich).

Andere Faktoren sind folglich alle anderen erklärenden Variablen wie Alter, Bildung, Anzahl an Kindern, Familienstand und Staatsbürgerschaft. Um die Difference in differences-Schätzung durchführen und die Schätzgleichung identifizieren zu können, muss die Common trend assumption erfüllt sein. Diese besagt, dass die Kontroll- und Treatmentgruppe vor der Reform einem gemeinsamen Langzeittrend folgen müssen, damit die gewählte Methode angewendet werden darf.

In Abbildung 4 werden die durchschnittlichen Wochenarbeitsstunden nach Gruppen aufgeschlüsselt über einen Zeitraum von 19 Jahren verglichen. Die Daten stammen wieder aus dem Mikrozensus, werden aber EU-weit in Eurostat unter dem European Labour Force Survey (EU-LFS) angeglichen (European Comission o. D.). Die Kurve der Kontrollgruppe mittelt die Werte für Wien, während die Kurve der Treatmentgruppe alle restlichen Bundesländer exklusive OÖ umfasst. Auf deskriptiver Ebene scheint die Common trend assumption erfüllt, da sich der Trend in Kontroll- und Treatmentgebieten bis zur Reform ähnlich verhält. ${ }^{8}$

\section{Ergebnisse}

Der Regressionsoutput in Tabelle 2 ist folgendermaßen aufgebaut: Die zugrunde liegenden Daten kommen, wie bereits beschrieben, aus der Mikrozensus-Erhebung und werden auf das für die Forschungsfrage geeignete Sample eingeschränkt: „Frauen mit Kindern

8 Es ist auf aggregiertem EU-Level nicht möglich nach Anzahl an Kindern zu filtern. Diese Grafik inkludiert alle Frauen im erwerbsfähigen Alter, die in den jeweiligen Bundesländern wohnhaft sind und eine wöchentliche Normalarbeitszeit in ihrem Hauptberuf angegeben haben. 
Zangerl, Pham: Die Auswirkungen von Kindergartengebühren auf die Erwerbstätigkeit von Frauen mit Kindern

Tabelle 2: OLS Regression Output Frauen mit Kindern unter sechs Jahren

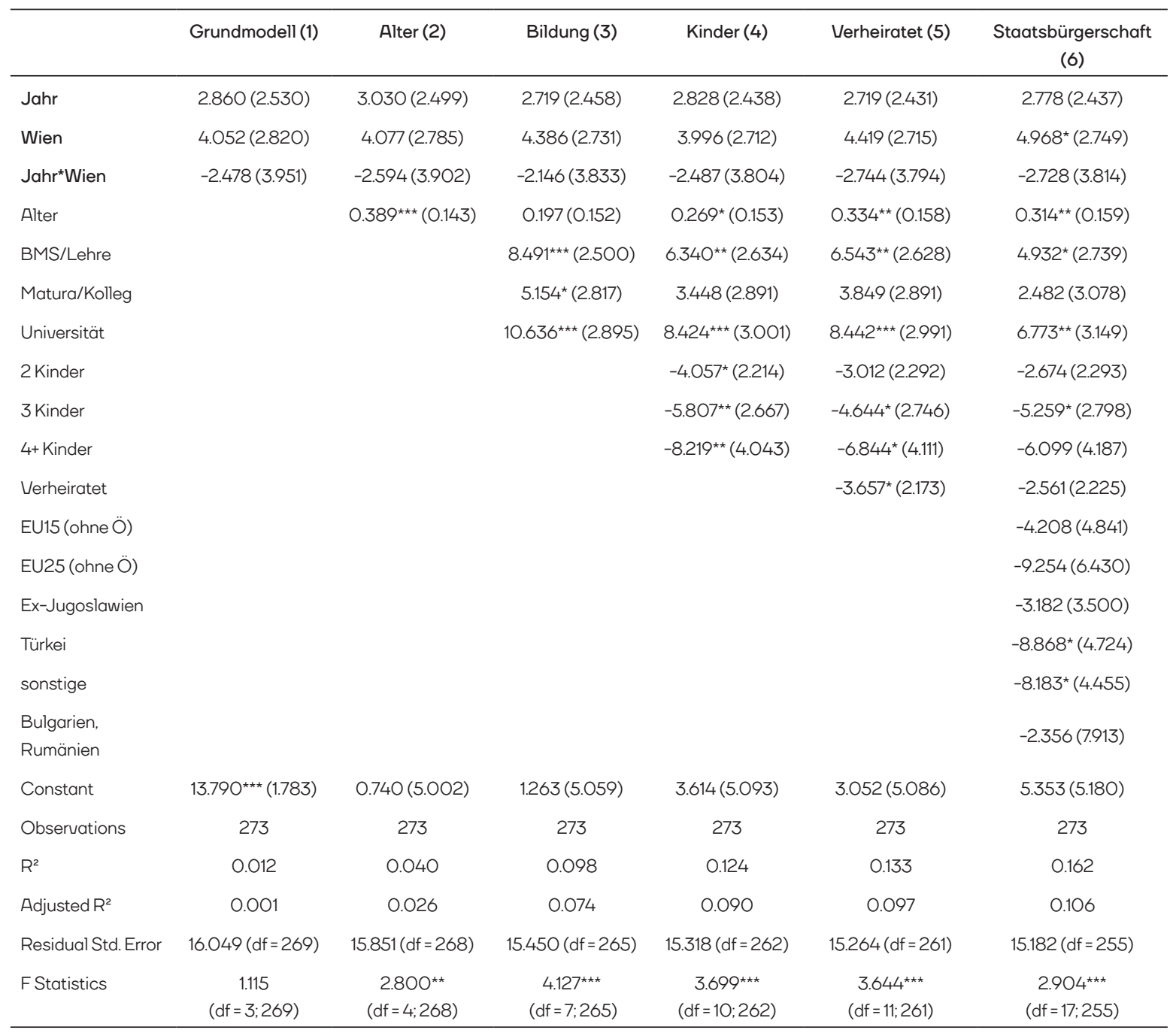

Quelle: Mikrozensus, eigene Berechnungen

$\star * * \quad$ Significant at the 1 percent level.

** Significant at the 5 percent level.

* Significant at the 10 percent level.

Die folgenden Dummyvariablen werden ggü. einer Baselinevariable interpretiert; Jahr: 2008, Wien: Restösterreich ohne OÖ, Bildung: Pflichtabschluss, Anzahl Kinder: 1 Kind, Verheiratet: Unverheiratet

unter sechs Jahren“. Die Ergebnisse aus der Tabelle lassen sich somit für Frauen mit Kindern unter sechs Jahren in Österreich interpretieren.

Die erste Spalte enthält die Beschriftungen der jeweiligen erklärenden Variablen aus Kapitel 5 - Daten und Variablen. Die zwei essenziellen Variablen Jahr und Wien für die Difference in differences-Schätzung sind hervorgehoben.

Das adjusted $R^{2}$ im Regressionsoutput beträgt 10,6 \% für das vollständige Modell. Das bedeutet, dass alle Variablen im Modell 10,6 \% der Variation in den Wochenarbeitsstunden erklären.
Der Standardfehler der Residuen (Residual Std. Error) wird zur Berechnung der adjusted $R^{2}$ und F-Statistik benötigt. Die F-Statistik resultiert aus dem F-Test, einem Signifikanztest, der auf die gemeinsame Signifikanz aller inkludierten Variablen testet. Dies hilft bei der Entscheidung der im Modell verwendeten Variablen. Die Nullhypothese lautet: Keine der inkludierten Variablen hat einen signifikanten Einfluss auf die abhängige Variable (Restringiertes Modell). Die Alternativhypothese lautet: Alle inkludierten Variablen haben gemeinsam einen signifikanten Einfluss auf die abhängige Variable. Kann die Nullhypothese nicht ver- 
worfen werden, ist noch nicht sicher, ob die Aufnahme dieser bestimmten Variablen im Modell sinnvoll für die Fragestellung ist. Die Inklusion der Variablen ist sinnvoll, wenn der p-Wert des F-Tests signifikant ausfällt (Wooldridge 2016: 143).

Die erste Zeile enthält die Modellnamen. Von links nach rechts wird in jeder Spalte eine zusätzliche Variable zur Schätzgleichung hinzugefügt.

Durch diese Methode lassen sich die Effekte einer bestimmten zusätzlichen Variable in der Schätzgleichung gut veranschaulichen. Zudem wird so auf Robustheit getestet, was bedeutet, dass sich die Schätzkoeffizienten derselben Variable in mehreren Modellen möglichst wenig stark voneinander unterscheiden sollen.

Wie im Regressionsoutput der Tabelle 2 zu sehen ist, weisen Frauen in Wien eine höhere Erwerbsbeteiligung auf als Frauen in Restösterreich. Sie arbeiten durchschnittlich 4,0 bis 5,0 Stunden mehr pro Woche. Diese erhöhte Erwerbsbeteiligung ist sogar auf dem 10 \%-Level signifikant, wenn die Variable Staatsbürgerschaft im Modell (6) aufgenommen wird. Ein auf $10 \%$ signifikanter Effekt bedeutet eine mögliche Annahme des gemessenen Effekts mit einer $10 \%$-Wahrscheinlichkeit zur Fehlerhaftigkeit. Das heißt, es kann mit einer Fehleranfälligkeit von $10 \%$ davon ausgegangen werden, dass sich die Anzahl der Wochenarbeitsstunden für Frauen mit Kindern in Wien von der Anzahl für Frauen mit Kindern in Restösterreich unterscheiden.

Der Interaktionsterm JahrWien stabilisiert für alle Modelle bei ca. 2,5 Wochenstunden. Dies ist gleichbedeutend mit einer Reduktion der durchschnittlichen Wochenarbeitszeit in Höhe von 2,5 Stunden in Folge der Abschaffung der Kindergartengebühren. Dies könnte ein Zeichen für den anfangs angesprochenen negativen Einkommenseffekt sein, bei dem die Frauen aufgrund der Erhöhung des verfügbaren Einkommens durch die Senkung der Kindergartengebühren eine Reallokation zwischen Arbeit und Freizeit hin zu Freizeit durchführen. Dieser Effekt ist jedoch nicht signifikant, weshalb die Nullhypothese, dass die Policy keinen Einfluss auf die Wochenarbeitsstunden habe, nicht verworfen werden kann.

Das Alter hat einen signifikant positiven Effekt in drei von fünf Modellen. Die höchsten Bildungsabschlüsse werden immer gegenüber der Basis (in diesem Fall Pflichtschulabschluss) interpretiert und kommen für die beiden Kategorien BMS/Lehre und Universität auf signifikant positive Ergebnisse. Für die Kategorie Matura ist das nur in einem von vier Fällen zutreffend.
Diese Ergebnisse gehen einher mit den Erwartungen aus der klassischen Humankapitaltheorie, die einen positiven Zusammenhang zwischen Bildung, Alter und Produktivität in Form von Arbeitsstunden herstellt.

Jedes zusätzliche Kind reduziert signifikant das Arbeitsaufkommen der Frau. Dies haben wir im Theorieteil bereits dem erhöhten Gesamtaufwand im Haushalt zugeordnet, der die Wahrscheinlichkeit reduziert, am Arbeitsmarkt zu partizipieren. Ebenso reduzieren verheiratete Frauen ihre Wochenarbeitszeit im Durchschnitt um 2,6 bis 3,7 Wochenstunden gegenüber nicht verheirateten Frauen, eine Ausprägung des Einflusses des vorherrschenden Familienmodells auf die Arbeitsmarktpartizipation der Frau.

Weitere signifikante Reduktionen der normalen Wochenarbeitsstunden treten durch die Inklusion der Variable Staatsbürgerschaft für Türkei $(-8,9)$ und die Sammelkategorie Sonstige $(-8,1)$ auf.

Zum Abschluss muss hier noch einmal ausdrücklich kritisch auf die Interpretation signifikanter und nicht signifikanter Effekte sowie auf die Richtung und Größe der Effekt hingewiesen werden. In erster Linie sind Wechselwirkungen in den Signifikanzen der Hinzufügung von Modellvariablen und der Variierung der Samplegröße zuzuordnen. Dies lässt noch keine Aussage darüber zu, wie gut dieses Modell tatsächlich Auswirkungen auf die normale Wochenarbeitszeit von Frauen mit Kindern abbildet. Denkbar sind nicht beachtete Variablen oder ökonomisch irrelevante, aber im Modell enthaltene Variablen, die einen Einfluss auf das Ergebnis haben.

\section{Eingrenzungen}

Die Weltwirtschaftskrise 2009 könnte eine wesentliche, in dieser Analyse nicht beachtete Rolle spielen. Für den Fall, dass sich die Krise unterschiedlich in Treatment- und Kontrollgruppe ausgewirkt hat, würde das die Ergebnisse verzerren.

Des Weiteren wurde eingangs auf wichtige institutionelle Bedingungen wie die Höhe der Kindergartengebühren und die Zugänglichkeit der Einrichtungen (Öffnungszeiten, Entfernung etc.) eingegangen, die nicht ins Modell miteingeflossen sind. Es steht außer Frage, dass eine detailliertere Erhebung zur Kinderanzahl präzisere Ergebnisse liefern könnte, indem Frauen mit Kindern unter drei Jahren vom Sample exkludiert werden, um frühkindliche Erziehung besser von Kindergarten unterscheiden zu können. 


\section{Conclusio}

Das Gesamtausmaß an geleisteten Wochenarbeitsstunden von Frauen mit Kindern unter sechs Jahren ist in Wien um 4,0 bis 4,9 Stunden höher als in Restösterreich. In den Ergebnissen lassen sich keine signifikanten Zusammenhänge mit der Befreiung von den Elternbeiträgen im Herbst 2009 herstellen. Dies ist sowohl Resultat der geringen Fallhöhe $(n=273)$ des Samples als auch dem höheren Ausgangsniveau in Wien geschuldet. Darüber hinaus werden andere, in der Forschung gut belegte Effekte sichtbar. Das vorgelegte Modell kann in Bezug auf Alter, Bildungsgrad, Anzahl an Kindern und Familienstand bestätigt werden und demonstriert, dass die theoretischen Überlegungen richtig waren.

Welche Richtung der Effekt der Gebührenabschaffung auf das Wochenarbeitsstundenausmaß von Frauen mit Kindern unter sechs Jahren hat, kann aufgrund dieser Analyse nicht klar gesagt werden. Weder die neoklassische Arbeitsmarkttheorie, die eine Reduktion der Arbeitsstunden nahelegt, wenn das verfügbare Einkommen steigt, noch die bestehende Forschung zu Reproduktionsarbeit und Frauenerwerbstätigkeit, wie u. a. Blau/Tekin (2007) und Brayfield (1995) vorführen, können hiermit widerlegt werden.

Es kann nicht angenommen werden, dass die Abschaffung - im gewissen Sinne Subvention von Kosten - deckungsgleiche Effekte wie die Einführung von Gebühren hat. Daher wäre es interessant, an die vorliegende Analyse mit neuen Daten aus dem Jahr 2019 anzuknüpfen, um sich die Effekte der Wiedereinführung der Kindergartengebühren in Oberösterreich anzusehen. Zusätzlich lassen sich aus einer länderübergreifenden Analyse mit deutlich mehr Beobachtungen sicherlich weitere Erkenntnisse gewinnen. Die Herausforderungen hierbei bestehen allerdings in der Harmonisierung der auch in der Arbeit genannten institutionellen Differenzen über den European Labour Force Survey hinweg.

Des Weiteren dürfen die qualitativen Dimensionen von Arbeit und Kinderbetreuung nicht außer Acht gelassen werden. Neben dem monetären Effekt auf Wochenarbeitsstunden spielen gerade im Elementarbereich institutionelle Faktoren wie Öffnungszeiten eine große Rolle: Kindergärten der Stadt Wien sind wochentags von 06:30 bis 17:30 Uhr geöffnet. $95 \%$ der öffentlichen Kindergärten sind weniger als 15 Tage im Jahr geschlossen. In den übrigen Bundesländern ist die
Verfügbarkeit mit über 36 Schließtagen pro Jahr und kürzeren Öffnungszeiten wesentlich begrenzter.

\section{Literatur}

Arbeiterkammer Oberösterreich (2018): Kinderbetreuung von A bis Z. Online: https://media.arbeiterkammer.at/ooe/ publikationen/berufundfamilie/B_2018_Kinderbetreuung_von_A_bis_Z.pdf [06.07.2018].

Berghammer, C. (2014): „The return of the male breadwinner model? Educational effects on parents' work arrangements in Austria, 1980-2009“. Work, Employment and Society, 28 (4), 611-632. DOI: 10.1177/0950017013500115.

Blau, D./Erdal, T. (2007): „The determinants and consequences of child care subsidies for single mothers in the USA“. Journal of Population Economics, (20) 4, 719-741.

Borjas, G. J./Van Ours, J. C. (200o): Labor economics. Bd. 2. Boston: McGraw-Hill.

Brayfield, A. P. (1995): „A Bargain At Any Price? Child Care Costs and Women's Employment". Social Science Research, 24 (2), 188-214. DOI: https://doi.org/10.1006/ ssre.1995.1007.

Dingeldey, I. (Hg., 200o): Erwerbstätigkeit und Familie in Steuer-und Sozialversicherungssystemen: Begünstigungen und Belastungen verschiedener familialer Erwerbsmuster im Ländervergleich. Opladen: Leske + Budrich.

European Commission (o. D.): Eurostat Database. Online: https://ec.europa.eu/eurostat/web/lfs/data/database?p_p_ id=NavTreeportletprod_WAR_ NavTreeportletprod_ INSTANCE_IFjhoVbmPFHt\&p_p_lifecycle $=0 \& p \_p \_$ state $=$ normal\&p_p_mode $=$ view\&p_p_col_id $=$ column2\&p_p_col_count $=1$ [05.07.2018].

Eurostat (2017): Beschäftigungsstatistik. Online: https:// ec.europa.eu/eurostat/statistics-explained/index.php/ Employment_statistics [05.09.2018].

- (2018): Degree of urbanisation classification - 2011 revision. Online: http://ec.europa.eu/eurostat/statistics-explained/index.php/Degree_of_urbanisation_classification_-_2011_revision [04.07.2018].

Fortin, N. M. (2005): „Gender Role Attitudes and the Labourmarket Outcomes of Women across OECD Countries". Oxford Review of Economic Policy, 21 (3), 416-438. DOI: 10.1093/oxrep/grio24.

Haider, A./Heineck, G./Neuwirth, N. (2004): Zwischen Reproduktions- und Erwerbsarbeit: Der Zusammenhang von Kinderbetreuung, Pflege und Frauenerwerbstätigkeit. Working Paper Nr. 42. Österreichisches Institut für Familienforschung. Online: https://www.oif.ac.at/fileadmin/user_upload/p_oif/Working_Paper/wp_42_reproduktion_erwerb.pdf [23.02.2020].

Kurier (2018): Kindergartengebühren in OÖ: 20 Prozent umoder abgemeldet. Online: https://kurier.at/politik/inland/ kindergartengebuehren-in-ooe-20-prozent-um-oderabgemeldet/400112225 [16.09.2018]. 
Land Oberösterreich (o. D.): Oö. KinderbetreuungsgesetzNovelle 2009. Online: https://www.land-oberoesterreich. gv.at/83758.htm [16.09.2018].

Matysiak, A. (2011): Interdependencies between fertility and women's labour supply. European studies of population 17. Dordrecht/New York: Springer.

Statistik Austria (2018a): Erwerbstätige. Online: https:// www.statistik.at/web_de/statistiken/menschen_und_ gesellschaft/arbeitsmarkt/erwerbstaetige/index.html [04.07.2018].

- (2018b): Mikrozensus ab 2004. Online: http://www.statistik. at/web_de/frageboegen/private_haushalte/mikrozensus/index.html [04.07.2018].

- (2018c): Teilzeit, Teilzeitquote. Online: https://www.statistik.at/web_de/statistiken/menschen_und_gesellschaft/ arbeitsmarkt/arbeitszeit/teilzeitarbeit_teilzeitquote/index.html [04.07.2018]
Wel, F. van/Knijn, T. (2006): „Transitional Phase or a New Balance?: Working and Caring by Mothers With Young Children in the Netherlands". Journal of Family Issues, 27 (5), 633-651. DOI: 10.1177/0192513X05284858.

Wooldridge, J. M. (2016): Introductory econometrics: a modern approach. Boston, MA: Cengage Learning.

\section{Appendix}

Abbildung 5: Abschaffung der Kindergartengebühren nach neoklassischer Arbeitsmarkttheorie

\section{FIGURE 2-7 The Effect of a Change in Nonlabor Income on Hours of Work}

An increase in nonlabor income leads to a parallel, upward shift in the budget line, moving the worker from point $P_{0}$ to point $P_{1}$. (a) If leisure is a normal good, hours of work fall. (b) If leisure is an inferior good, hours of work increase.

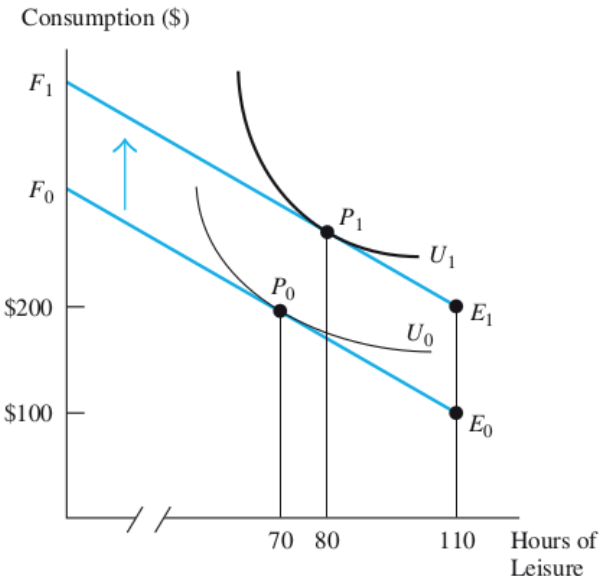

(a) Leisure Is a Normal Good

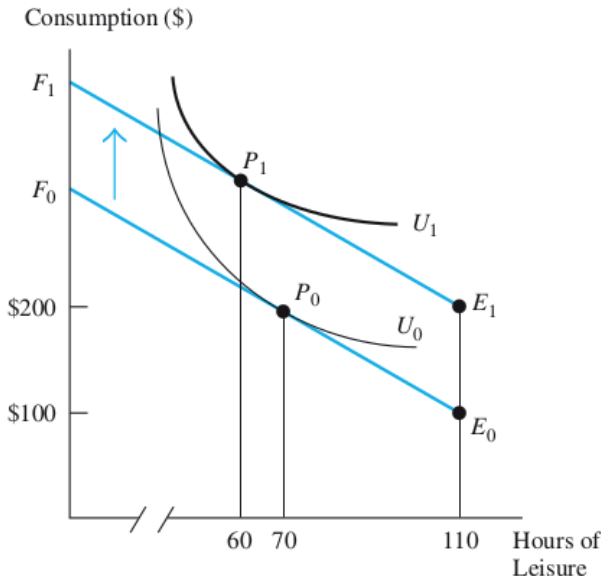

(b) Leisure Is an Inferior Good

Quelle: Wooldridge 2016 